\title{
QUANTIZATION OF NON-UNITARY GEOMETRIC CLASSICAL $r$-MATRICES
}

\author{
P. Etingof \& M. Graña
}

\begin{abstract}
In this paper we explicitly attach to a geometric classical r-matrix $r$ (not necessarily unitary), a geometric (i.e., set-theoretical) quantum R-matrix $R$, which is a quantization of $r$. To accomplish this, we use the language of bijective cocycle 7-tuples, developed by A. Soloviev in the study of set-theoretical quantum R-matrices. Namely, we define a classical version of bijective cocycle 7-tuples, and show that there is a bijection between them and geometric classical r-matrices. Then we show how any classical bijective cocycle 7-tuple can be quantized, and finally use Soloviev's construction, which turns a (quantum) bijective cocycle 7tuple into a geometric quantum R-matrix.
\end{abstract}

\section{Introduction}

Let $X$ be a smooth affine algebraic variety over $\mathbb{C}$. A formal diffeomorphism of $X$ is an automorphism of the ring $\mathcal{O}[X][[\hbar]]$ which is the identity modulo $\hbar$. That is, it looks like $1+\hbar r+O\left(\hbar^{2}\right)$. Note that for such a map to be a ring homomorphism, $r$ must be a derivation, i.e., $r \in \operatorname{Vect}(X)$.

A geometric solution of the Quantum Yang-Baxter Equation (QYBE) is a formal diffeomorphism $R$ of $X \times X$ such that $R^{12} R^{13} R^{23}=R^{23} R^{13} R^{12}$ as formal diffeomorphisms of $X \times X \times X$. It is straightforward to check that if $R=1+\hbar r+$ $O\left(\hbar^{2}\right)$ is a geometric solution of the QYBE, then $r$ is a geometric solution of the Classical Yang-Baxter Equation (CYBE), i.e. $\left[r^{12}, r^{13}\right]+\left[r^{12}, r^{23}\right]+\left[r^{13}, r^{23}\right]=0$. The well known quantization problem is: given a geometric solution $r$ of the CYBE, construct a geometric solution $R$ of the QYBE which restricts to $r$ in degree 1 , i.e., $R=1+\hbar r+O\left(\hbar^{2}\right)$. Such an $R$ is called a geometric quantization of $r$.

In $[\mathrm{EK}]$ it is proved that any solution of CYBE can be quantized. However, the proof does not give a simple explicit formula for $R$, and furthermore it is not clear if the map $R$ is geometric when $r$ is. On the other hand, in [ES], the problem of geometric quantization is solved for unitary r-matrices (i.e., satisfying the unitarity condition $\left.r^{21}=-r\right)$ : it is shown that in this case the geometric quantization $R$ exists and satisfies the quantum unitarity condition $R^{21} R=1$. For this, using the approach of [ESS], it is shown that both classical and quantum

Received December 13, 2002.

The work of P.E. was supported by the NSF grant DMS-9988796. The work of M.G. was supported by Conicet. 
geometric r-matrices are parametrized by some group-theoretical data, at the level of which quantization basically reduces to the usual exponential map.

In this paper we generalize the result of [ES] to the non-unitary case. Namely, we prove the following result.

Theorem 1.1. Any geometric solution to the CYBE admits a geometric quantization.

As in the unitary case, this is accomplished using the group-theoretical approach, developed in [S]. In this sense, this paper completes the square

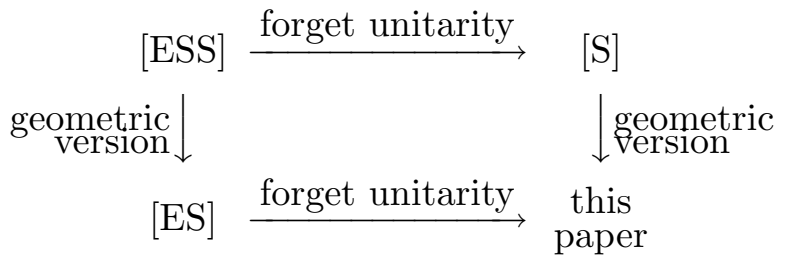

\section{Cocycle 7-tuples}

Recall the following definition from $[\mathrm{S}]$.

Definition 2.1. A bijective cocycle 7-tuple (BCST) is a 7-tuple

$$
\left(G, A, X, \rho_{G A}, \rho_{G A X}, \pi, \Psi\right),
$$

where $G$ and $A$ are groups, $X$ a set, $\rho_{G A}$ is an action of $G$ on $A, \rho_{G A X}$ is an action of $A \rtimes G$ on $X, \pi: G \rightarrow A$ is a bijective 1-cocycle (i.e., $\pi(g h)=\pi(g)(g \pi(h)))$ and $\Psi: X \rightarrow A$ is an $A \rtimes G$-equivariant map (with $A$ acting on itself by conjugation) whose image generates $A$.

A BCST gives two actions of $G$ on $X$, given by composing $\rho_{G A X}$ with the inclusion $G \rightarrow A \rtimes G$ and with the map $g \mapsto(\pi(g), g)$. Thus, it defines a map $G \rightarrow \mathbb{S}_{X} \times \mathbb{S}_{X}$ (where $\mathbb{S}_{X}$ is the group of bijections $X \rightarrow X$ ). The BCST is said to be faithful if this map is injective.

We state a result from [S], slightly modified to fit our definition of 1-cocycles:

Lemma 2.2. Let $\left(G, A, X, \rho_{G A}, \rho_{G A X}, \pi, \Psi\right)$ be a BCST. Let $R: X \times X \rightarrow$ $X \times X$ be defined by

$$
R(x, y)=(x \check{*} y, x \check{y} y)
$$

where

$$
x \check{*} y=\rho_{G A X}\left(\pi^{-1}\left(y^{-1}\right)\right)(x) \text { and } x \text { ó } y=\rho_{G A X}\left(\pi^{-1}\left((x \check{*} y)^{-1}\right)\right)^{-1}\left(\rho_{G A X}(x \check{*} y)(y)\right)
$$

(we write here, for $z \in X, z$ instead of $\Psi(z) \in A$ for not overcharging the notation). Then $R$ is a set theoretical solution of the braid equation, i.e.,

$$
R^{12} R^{13} R^{23}=R^{23} R^{13} R^{12} .
$$


In fact, the solution (2.3) is also non-degenerate in the sense of $[\mathrm{S}]$. The main result of $[\mathrm{S}]$ is that formula (2.3) defines a bijection (or, more precisely, a categorical equivalence) between faithful bijective cocycle 7-tuples and nondegenerate set-theoretical solutions of QYBE.

Let us now generalize this theory to the quasiclassical situation. Our ground field will always be $\mathbb{C}$.

Definition 2.4. A classical bijective cocycle 7-tuple (CBCST) is a 7-tuple

$$
\left(\mathfrak{g}, \mathfrak{a}, X, \rho_{\mathfrak{g a}}, \rho_{\mathfrak{g a} X}, \pi, \Psi\right),
$$

where $\mathfrak{g}$ and $\mathfrak{a}$ are Lie algebras, $X$ a smooth affine algebraic variety, $\rho_{\mathfrak{g a}}$ is an action of $\mathfrak{g}$ on $\mathfrak{a}, \rho_{\mathfrak{g} \mathfrak{a} X}$ is an action of $\mathfrak{a} \rtimes \mathfrak{g}$ on $X, \pi: \mathfrak{g} \rightarrow \mathfrak{a}$ is a bijective (non-commutative) 1-cocycle and $\Psi: X \rightarrow \mathfrak{a}$ is an $\mathfrak{a} \rtimes \mathfrak{g}$-equivariant map (with $\mathfrak{a}$ acting on itself by commutator) whose image generates $\mathfrak{a}$.

A CBCST gives two actions of $\mathfrak{g}$ on $X$, given by composing $\rho_{\mathfrak{g a} X}$ with the inclusion $\mathfrak{g} \rightarrow \mathfrak{a} \rtimes \mathfrak{g}$ and with the map $g \mapsto(\pi(g), g)$. Thus, it defines a map $\mathfrak{g} \rightarrow \operatorname{Vect}(X) \times \operatorname{Vect}(X)$. The CBCST is said to be faithful if this map is injective.

The first part of the proof of Theorem 1.1 is to relate classical bijective cocycle 7-tuples and geometric solutions of the CYBE. One can consider the categories of CBCST's and of geometric solutions of the CYBE, with the obvious notion of morphisms in both.

Theorem 2.5. There is a an equivalence of categories between faithful CBCST's and geometric solutions of the CYBE.

Proof. We will construct mutually (quasi)inverse functors in both directions. More precisely, we will do this only for objects, as the extension to morphisms is straightforward (and not used). In the proof, we will refer to a sequence of lemmas, which are stated and proved in $\S 3$.

Let $r$ be a geometric solution of the CYBE. Then we have

$$
\begin{aligned}
& r \in \operatorname{Vect}(X) \otimes \mathcal{O}(X) \oplus \mathcal{O}(X) \otimes \operatorname{Vect}(X), \\
& r=\sum_{i} a_{i}^{1} \otimes a_{i}^{0}+\sum_{j} b_{j}^{0} \otimes b_{j}^{1}, \\
& {\left[r^{12}, r^{13}\right]+\left[r^{12}, r^{23}\right]+\left[r^{13}, r^{23}\right]=0 .}
\end{aligned}
$$

We will omit the summation sign in expressions of the type $\sum_{i} a_{i}^{1} \otimes a_{i}^{0}$ or $\sum_{j} b_{j}^{0} \otimes b_{j}^{1}$. By looking at the degree of components, we can split the CYBE into three equations as follows:

$$
\begin{aligned}
& 0=\left[a_{i}^{1}, a_{k}^{1}\right] \otimes a_{i}^{0} \otimes a_{k}^{0}-a_{i}^{1} \otimes a_{k}^{1} \cdot a_{i}^{0} \otimes a_{k}^{0}-a_{i}^{1} \otimes b_{l}^{0} \otimes b_{l}^{1} \cdot a_{i}^{0}, \\
& 0=-a_{k}^{1} \cdot b_{j}^{0} \otimes b_{j}^{1} \otimes a_{k}^{0}+b_{j}^{0} \otimes\left[b_{j}^{1}, a_{k}^{1}\right] \otimes a_{k}^{0}+b_{j}^{0} \otimes a_{k}^{1} \otimes b_{j}^{1} \cdot a_{k}^{0}, \\
& 0=a_{i}^{1} \cdot b_{l}^{0} \otimes a_{i}^{0} \otimes b_{l}^{1}+b_{j}^{0} \otimes b_{j}^{1} \cdot b_{l}^{0} \otimes b_{l}^{1}+b_{j}^{0} \otimes b_{l}^{0} \otimes\left[b_{j}^{1}, b_{l}^{1}\right] .
\end{aligned}
$$

We define $\mathfrak{g}_{1}=\operatorname{span}\left\{a_{i}^{1}\right\}_{i}, \mathfrak{g}_{2}=\operatorname{span}\left\{b_{j}^{1}\right\}_{j}$ (we assume that the expression (2.6) has the minimal possible number of summands). It is easy to see from (2.7) 
and (2.9) that both $\mathfrak{g}_{i}$ 's are Lie subalgebras of $\operatorname{Vect}(X)$. Also, $\left[\mathfrak{g}_{1}, \mathfrak{g}_{2}\right] \subseteq \mathfrak{g}_{1}+\mathfrak{g}_{2}$ by $(2.8)$. For $x \in \mathcal{O}(X)^{*}$, let

$$
p(x)=\left(-x\left(a_{i}^{0}\right) a_{i}^{1}, x\left(b_{j}^{0}\right) b_{j}^{1}\right) \in \mathfrak{g}_{1} \oplus \mathfrak{g}_{2},
$$

and call $\mathfrak{g}=\operatorname{Im}(p)$. It is proved in (3.2) below that $\mathfrak{g}$ is a Lie subalgebra of $\mathfrak{g}_{1} \oplus \mathfrak{g}_{2}$.

We define $V_{1}, V_{2} \subset \mathcal{O}(X)$ as $V_{1}=\operatorname{span}\left\{a_{i}^{0}\right\}_{i}, V_{2}=\operatorname{span}\left\{b_{j}^{0}\right\}_{j}$, and we put $\mathfrak{a}=\left(V_{1}+V_{2}\right)^{*}$. We can restrict $p$ to $\mathfrak{a}$ and we get an isomorphism $\left.p\right|_{\mathfrak{a}}: \mathfrak{a} \rightarrow \mathfrak{g}$. Call $\pi=\left.p\right|_{\mathfrak{a}} ^{-1}$.

Consider the action of $\operatorname{Vect}(X)$ on $\mathcal{O}(X)$. From $(2.7),(2.8),(2.9)$ one can see that

$$
\begin{aligned}
& \mathfrak{g}_{1} \cdot V_{1} \subset V_{1}+V_{2}, \quad \mathfrak{g}_{1} \cdot V_{2} \subset V_{2}, \\
& \mathfrak{g}_{2} \cdot V_{1} \subset V_{1}, \quad \mathfrak{g}_{2} \cdot V_{2} \subset V_{1}+V_{2} .
\end{aligned}
$$

We denote by $*$ and $\_$the left and right actions of $\mathfrak{g}$ on $\mathfrak{a}$ via projections to the first and second coordinates. We also use this notation for the maps $\mathfrak{a} \times \mathfrak{a} \rightarrow \mathfrak{a}$ obtained by composing these actions with $p$ (we warn that these maps are not actions since $p$ is not a Lie algebra homomorphism). Specifically,

$$
\begin{aligned}
& (x * y)(f)=(p(x) * y)(f)=-x\left(a_{i}^{0}\right)\left(a_{i}^{1} \cdot y\right)(f)=x\left(a_{i}^{0}\right) y\left(a_{i}^{1} \cdot f\right), \\
& (x \multimap y)(f)=(x \multimap p(y))(f)=y\left(b_{j}^{0}\right)\left(x \cdot b_{j}^{1}\right)(f)=y\left(b_{j}^{0}\right) x\left(b_{j}^{1} \cdot f\right) .
\end{aligned}
$$

Using these actions and the bijection $\pi$ we equip $\mathfrak{a}$ with a Lie algebra structure:

$$
[x, y]=-x * y+y * x+\pi([p(x), p(y)]) .
$$

We have to prove that this is indeed a Lie algebra structure, which we do in Lemma 3.1 below. Then $\pi$ is automatically a 1-cocycle.

We prove in Lemma 3.5 that the action $*$ of $\mathfrak{g}$ on $\mathfrak{a}$ is by derivations (i.e., * $: \mathfrak{g} \rightarrow \operatorname{Der}(\mathfrak{a}))$. This allows us to take the semidirect product $\mathfrak{a} \rtimes \mathfrak{g}$, whose structure, we recall, is

$$
[(a, g),(b, h)]=(g * b-h * a+[a, b],[g, h]) .
$$

Notice from (3.3) below that the Lie algebra structure in $\mathfrak{a}$ is

$$
[x, y]=-y\left(a_{i}^{0}\right)\left(a_{i}^{1} \cdot x\right)-y\left(b_{j}^{0}\right)\left(b_{j}^{1} \cdot x\right)=x\left(a_{i}^{0}\right)\left(a_{i}^{1} \cdot y\right)+x\left(b_{j}^{0}\right)\left(b_{j}^{1} \cdot y\right) .
$$

Consider the action of $\mathfrak{g}$ on $X$ given by the projection to the first coordinate, i.e.

$$
\rho(p(x))=-x\left(a_{i}^{0}\right) a_{i}^{1} .
$$

Let $d: \mathfrak{g} \rightarrow \mathfrak{g}_{1}+\mathfrak{g}_{2}, d\left(g_{1}, g_{2}\right)=g_{1}-g_{2}$. Define $\rho_{\mathfrak{a} X}: \mathfrak{a} \rightarrow \operatorname{Vect}(X)$ as follows:

$\rho=\left(\mathfrak{a} \stackrel{-p}{\longrightarrow} \mathfrak{g} \stackrel{d}{\longrightarrow} \mathfrak{g}_{1}+\mathfrak{g}_{2} \subset \operatorname{Vect}(X)\right), \quad$ i.e. $\rho(x)=x\left(a_{i}^{0}\right) a_{i}^{1}+x\left(b_{j}^{0}\right) b_{j}^{1}$. 
We prove in Lemma 3.6 that this is a map of Lie algebras. We can lift this action to $\mathfrak{a} \rtimes \mathfrak{g}$ : define

$$
\rho_{\mathfrak{g} \mathfrak{a} X}: \mathfrak{a} \rtimes \mathfrak{g} \rightarrow \operatorname{Vect} X, \quad \rho(y, p(x))=y\left(a_{i}^{0}\right) a_{i}^{1}+y\left(b_{j}^{0}\right) b_{j}^{1}-x\left(a_{k}^{0}\right) a_{k}^{1} .
$$

From the structure of $\mathfrak{a} \rtimes \mathfrak{g}$ we have $[(0, p(x)),(y, 0)]=(x * y, 0)$. Let us check that this is indeed an action. We have

$$
\begin{aligned}
\rho(x * y)= & (x * y)\left(a_{i}^{0}\right) a_{i}^{1}+(x * y)\left(b_{j}^{0}\right) b_{j}^{1} \\
= & x\left(a_{k}^{0}\right) y\left(a_{k}^{1} \cdot a_{i}^{0}\right) a_{i}^{1}+x\left(a_{k}^{0}\right) y\left(a_{k}^{1} \cdot b_{j}^{0}\right) b_{j}^{1}, \\
{\left[\rho(p(x)), \rho_{\mathfrak{a} X}(y)\right]=} & {\left[-x\left(a_{k}^{0}\right) a_{k}^{1}, y\left(a_{i}^{0}\right) a_{i}^{1}+y\left(b_{j}^{0}\right) b_{j}^{1}\right] } \\
= & x\left(a_{k}^{0}\right) y\left(a_{i}^{0}\right)\left[a_{i}^{1}, a_{k}^{1}\right]-x\left(a_{k}^{0}\right) y\left(b_{j}^{0}\right)\left[a_{k}^{1}, b_{j}^{1}\right] \\
= & y\left(a_{k}^{1} \cdot a_{i}^{0}\right) x\left(a_{k}^{0}\right) a_{i}^{1}+y\left(b_{l}^{0}\right) x\left(b_{l}^{1} \cdot a_{i}^{0}\right) a_{i}^{1} \\
& \quad+y\left(a_{k}^{1} \cdot b_{j}^{0}\right) x\left(a_{k}^{0}\right) b_{j}^{1}-y\left(b_{j}^{0}\right) x\left(b_{j}^{1} \cdot a_{k}^{0}\right) a_{k}^{1} \\
= & \rho(x * y),
\end{aligned}
$$

as desired.

Define $\Psi_{\mathfrak{a}}: X \rightarrow \mathfrak{a}$ by restriction: $\Psi_{\mathfrak{a}}(x)(f)=f(x)$ for $f \in V$. Comparing (2.13) with (2.15) and (2.10) with (2.14), it is clear that $\Psi_{\mathfrak{a}}$ is $\mathfrak{a} \rtimes \mathfrak{g}$-invariant, i.e., $\left.\Psi_{\mathfrak{a} *}\right|_{x} \rho_{\mathfrak{g a} X}(a, g)=-(a, g) \cdot \Psi_{\mathfrak{a}}(x)=-\left[a, \Psi_{\mathfrak{a}}(x)\right]-g * \Psi_{\mathfrak{a}}(x)$. We have thus constructed a CBCST $\left(\mathfrak{g}, \mathfrak{a}, X, \rho_{\mathfrak{g a}}, \rho_{\mathfrak{g a} X}, \pi, \Psi_{\mathfrak{a}}\right)$.

Now we begin with a 7 -tuple $\left(\mathfrak{g}, \mathfrak{a}, X, \rho_{\mathfrak{g a}}, \rho_{\mathfrak{g a} X}, \pi, \Psi_{\mathfrak{a}}\right)$ and aim to construct $r$. First, consider the maps

$$
\begin{aligned}
& \alpha=X \stackrel{\Psi_{\mathfrak{a}}}{\longrightarrow} \mathfrak{a} \stackrel{-\pi^{-1}}{\longrightarrow} \mathfrak{g} \stackrel{i}{\longrightarrow} \mathfrak{a} \rtimes \mathfrak{g} \stackrel{\rho}{\longrightarrow} \text { Vect } X, \\
& \beta=X \stackrel{\Psi_{\mathfrak{a}}}{\longrightarrow} \mathfrak{a} \stackrel{\pi^{-1} \times \mathrm{id}}{\longrightarrow} \mathfrak{a} \rtimes \mathfrak{g} \stackrel{\rho}{\longrightarrow} \text { Vect } X .
\end{aligned}
$$

We call $\mathfrak{g}_{1}=\operatorname{Im}(\alpha), \mathfrak{g}_{2}=\operatorname{Im}(\beta)$. Now, $\alpha$ gives by composition a map $\mathfrak{g}_{1}^{*} \rightarrow$ $\mathcal{O}(X)$, which in turn is an element of $\mathfrak{g}_{1} \otimes \mathcal{O}(X)$. Analogously, $\beta$ gives a map $\mathfrak{g}_{2}^{*} \rightarrow \mathcal{O}(X)$, and in turn an element of $\mathcal{O}(X) \otimes \mathfrak{g}_{2}$. Call these elements $r^{1}, r^{2}$ respectively. We view them as elements of $\operatorname{Vect}(X) \otimes \mathcal{O}(X)$ and $\mathcal{O}(X) \otimes \operatorname{Vect}(X)$. Call

$$
r=r^{1}+r^{2}
$$

We prove in Lemma 3.7 that $r$ is a geometric solution of the CYBE. It is easy to see that both constructions

Geometric solutions of CYBE $\leadsto$ Classical bijective cocycle 7-tuples are inverse to each other. The condition on $\Psi(X)$ to generate $\mathfrak{a}$ guarantees that after applying CBCST $\rightsquigarrow$ Geom. sol. of CYBE $\rightsquigarrow$ CBCST one gets an algebra $\mathfrak{a}$ isomorphic to the original one. Analogously, the faithfulness condition guarantees that one recovers an algebra $\mathfrak{g}$ isomorphic to the original one. 


\section{Auxiliary lemmas}

Lemma 3.1. The definition in (2.12) equips $\mathfrak{a}$ with the structure of a Lie algebra.

Proof. The bilinearity and antisymmetry of the bracket are clear. Let us prove that it satisfies the Jacobi identity. We have

$$
\begin{aligned}
p(x * y) & =\left(-(x * y)\left(a_{i}^{0}\right) a_{i}^{1},(x * y)\left(b_{j}^{0}\right) b_{j}^{1}\right) \\
& =\left(-x\left(a_{k}^{0}\right) y\left(a_{k}^{1} \cdot a_{i}^{0}\right) a_{i}^{1}, x\left(a_{k}^{0}\right) y\left(a_{k}^{1} \cdot b_{j}^{0}\right) b_{j}^{1}\right), \\
p(x \multimap y) & =\left(-(x \multimap y)\left(a_{i}^{0}\right) a_{i}^{1},(x \multimap y)\left(b_{j}^{0}\right) b_{j}^{1}\right) \\
& =\left(-y\left(b_{l}^{0}\right) x\left(b_{l}^{1} \cdot a_{i}^{0}\right) a_{i}^{1}, y\left(b_{l}^{0}\right) x\left(b_{l}^{1} \cdot b_{j}^{0}\right) b_{j}^{1}\right), \\
{[p(x), p(y)] } & =\left[\left(-x\left(a_{i}^{0}\right) a_{i}^{1}, x\left(b_{j}^{0}\right) b_{j}^{1}\right),\left(-y\left(a_{k}^{0}\right) a_{k}^{1}, y\left(b_{l}^{0}\right) b_{l}^{1}\right)\right] \\
& =\left(\left[x\left(a_{i}^{0}\right) a_{i}^{1}, y\left(a_{k}^{0}\right) a_{k}^{1}\right],\left[x\left(b_{j}^{0}\right) b_{j}^{1}, y\left(b_{l}^{0}\right) b_{l}^{1}\right]\right) \\
& =\left(x\left(a_{i}^{0}\right) y\left(a_{k}^{0}\right)\left[a_{i}^{1}, a_{k}^{1}\right], x\left(b_{j}^{0}\right) y\left(b_{l}^{0}\right)\left[b_{j}^{1}, b_{l}^{1}\right]\right) \\
& =\left(-x\left(a_{k}^{0}\right) y\left(a_{i}^{0}\right)\left[a_{i}^{1}, a_{k}^{1}\right],-x\left(b_{l}^{0}\right) y\left(b_{j}^{0}\right)\left[b_{j}^{1}, b_{l}^{1}\right]\right),
\end{aligned}
$$

whence, using (2.7) and (2.9),

$$
\begin{aligned}
{[p(x), p(y)] } & =p(x * y)+p(x \circ y), \quad \text { i.e. } \\
{[x, y] } & =x \circ y+y * x .
\end{aligned}
$$

Notice that (3.2) proves that $\mathfrak{g}$ is a subalgebra of $\mathfrak{g}_{1} \oplus \mathfrak{g}_{2}$. Similarly we compute for $f \in \mathcal{O}(X)$,

$$
\begin{aligned}
& ((z * y) \multimap x)(f)=x\left(b_{j}^{0}\right)(z * y)\left(b_{j}^{1} \cdot f\right)=x\left(b_{j}^{0}\right) y\left(a_{k}^{1} b_{j}^{1} \cdot f\right) z\left(a_{k}^{0}\right), \\
& (z *(y \multimap x))(f)=z\left(a_{i}^{0}\right)(y \multimap x)\left(a_{i}^{1} \cdot f\right)=x\left(b_{l}^{0}\right) y\left(b_{l}^{1} a_{i}^{1} \cdot f\right) z\left(a_{i}^{0}\right), \\
& ((z \multimap x) * y)(f)=(z \multimap x)\left(a_{i}^{0}\right) y\left(a_{i}^{1} \cdot f\right)=x\left(b_{l}^{0}\right) y\left(a_{i}^{1} \cdot f\right) z\left(b_{l}^{1} \cdot a_{i}^{0}\right), \\
& (y \multimap(z * x))(f)=(z * x)\left(b_{j}^{0}\right) y\left(b_{j}^{1} \cdot f\right)=x\left(a_{k}^{1} \cdot b_{j}^{0}\right) y\left(b_{j}^{1} \cdot f\right) z\left(a_{k}^{0}\right) .
\end{aligned}
$$

Using (2.8) we get

$((z * y) \multimap x)-(z *(y \multimap x))-((z \multimap x) * y)+(y \multimap(z * x))=0$,

and using now (3.2), we have

$$
\begin{aligned}
& \pi[p(z * y), p(x)]-((z * y) * x)-(z * \pi[p(y), p(x)])+(z *(y * x)) \\
& \quad-(\pi[p(z), p(x)] * y)+((z * x) * y)+\pi[p(y), p(z * x)]-(y *(z * x))=0 .
\end{aligned}
$$


Now, we compute

$$
\begin{aligned}
{[x,[y, z]]=} & -x *[y, z]+[y, z] * x+\pi[p(x), p([y, z])] \\
= & x *(y * z)-x *(z * y)-x *(\pi[p(y), p(z)])-(y * z) * x \\
& +(z * y) * x+\pi[p(y), p(z)] * x-\pi[p(x), p(y * z)] \\
& +\pi[p(x), p(z * y)]+\pi[p(x),[p(y), p(z)]], \\
{[y,[z, x]]=} & y *(z * x)-y *(x * z)-y *(\pi[p(z), p(x)]) \\
& -(z * x) * y+(x * z) * y+\pi[p(z), p(x)] * y \\
& -\pi[p(y), p(z * x)]+\pi[p(y), p(x * z)]+\pi[p(y),[p(z), p(x)]], \\
{[z,[x, y]]=} & z *(x * y)-z *(y * x)-z *(\pi[p(x), p(y)]) \\
& -(x * y) * z+(y * x) * z+\pi[p(x), p(y)] * z \\
& -\pi[p(z), p(x * y)]+\pi[p(z), p(y * x)]+\pi[p(z),[p(x), p(y)]] .
\end{aligned}
$$

Applying (3.4) three times and Jacobi identity in $\mathfrak{g}$ we get the Jacobi identity in $\mathfrak{a}$.

Lemma 3.5. The algebra $\mathfrak{g}$ acts on $\mathfrak{a}$ by derivations with the action $*$ defined in (2.10).

Proof. This is straightforward: we compute

$$
\begin{aligned}
p(x *[y, z])= & p(x *(z * y)+x *(y \multimap z)) \\
= & \left(-x\left(a_{i}^{0}\right)(z * y+y \multimap z)\left(a_{i}^{1} \cdot a_{k}^{0}\right) a_{k}^{1}, x\left(a_{i}^{0}\right)(z * y+y \multimap z)\left(a_{i}^{1} \cdot b_{l}^{0}\right) b_{l}^{1}\right) \\
= & \left(-x\left(a_{i}^{0}\right) z\left(a_{m}^{0}\right) y\left(a_{m}^{1} a_{i}^{1} \cdot a_{k}^{0}\right) a_{k}^{1}-x\left(a_{i}^{0}\right) z\left(b_{n}^{0}\right) y\left(b_{n}^{1} a_{i}^{1} \cdot a_{k}^{0}\right) a_{k}^{1},\right. \\
& \left.x\left(a_{i}^{0}\right) z\left(a_{m}^{0}\right) y\left(a_{m}^{1} a_{i}^{1} \cdot b_{l}^{0}\right) b_{l}^{1}+x\left(a_{i}^{0}\right) z\left(b_{n}^{0}\right) y\left(b_{n}^{1} a_{i}^{1} \cdot b_{l}^{0}\right) b_{l}^{1}\right), \\
p(-[x * y, z])= & ((x * y) \multimap z)\left(a_{k}^{0}\right) a_{k}^{1}+(z *(x * y))\left(a_{k}^{0}\right) a_{k}^{1}, \\
& \left.\quad-((x * y)-z)\left(b_{l}^{0}\right) b_{l}^{1}-(z *(x * y))\left(b_{l}^{0}\right) b_{l}^{1}\right) \\
= & \left(z\left(b_{j}^{0}\right)(x * y)\left(b_{j}^{1} \cdot a_{k}^{0}\right) a_{k}^{1}+z\left(a_{i}^{0}\right)(x * y)\left(a_{i}^{1} \cdot a_{k}^{0}\right) a_{k}^{1},\right. \\
& \left.\quad-z\left(b_{j}^{0}\right)(x * y)\left(b_{j}^{1} \cdot b_{l}^{0}\right) b_{l}^{1}-z\left(a_{i}^{0}\right)(x * y)\left(a_{i}^{1} \cdot b_{l}^{0}\right) b_{l}^{1}\right) \\
= & \left(z\left(b_{j}^{0}\right) x\left(a_{m}^{0}\right) y\left(a_{m}^{1} b_{j}^{1} a_{k}^{0}\right) a_{k}^{1}+z\left(a_{i}^{0}\right) x\left(a_{m}^{0}\right) y\left(a_{m}^{1} a_{i}^{1} a_{k}^{0}\right) a_{k}^{1},\right. \\
& \left.\quad-z\left(b_{j}^{0}\right) x\left(a_{m}^{0}\right) y\left(a_{m}^{1} b_{j}^{1} b_{l}^{0}\right) b_{l}^{1}-z\left(a_{i}^{0}\right) x\left(a_{m}^{0}\right) y\left(a_{m}^{1} a_{i}^{1} b_{l}^{0}\right) b_{l}^{1}\right), \\
p(-[y, x * z])= & \left((y-(x * z))\left(a_{k}^{0}\right) a_{k}^{1}+((x * z) * y)\left(a_{k}^{0}\right) a_{k}^{1},\right. \\
& \left.\quad-(y \multimap(x * z))\left(b_{l}^{0}\right) b_{l}^{1}-((x * z) * y)\left(b_{l}^{0}\right) b_{l}^{1}\right) \\
= & \left((x * z)\left(b_{j}^{0}\right) y\left(b_{j}^{1} \cdot a_{k}^{0}\right) a_{k}^{1}+(x * z)\left(a_{i}^{0}\right) y\left(a_{i}^{1} \cdot a_{k}^{0}\right) a_{k}^{1},\right. \\
& \left.\quad-(x * z)\left(b_{j}^{0}\right) y\left(b_{j}^{1} \cdot b_{l}^{0}\right) b_{l}^{1}-(x * z)\left(a_{i}^{0}\right) y\left(a_{i}^{1} \cdot b_{l}^{0}\right) b_{l}^{1}\right) \\
= & \left(x\left(a_{m}^{0}\right) z\left(a_{m}^{1} \cdot b_{j}^{0}\right) y\left(b_{j}^{1} \cdot a_{k}^{0}\right) a_{k}^{1}+x\left(a_{m}^{0}\right) z\left(a_{m}^{1} \cdot a_{i}^{0}\right) y\left(a_{i}^{1} \cdot a_{k}^{0}\right) a_{k}^{1},\right. \\
& \left.\quad-x\left(a_{m}^{0}\right) z\left(a_{m}^{1} \cdot b_{j}^{0}\right) y\left(b_{j}^{1} \cdot b_{l}^{0}\right) b_{l}^{1}-x\left(a_{m}^{0}\right) z\left(a_{m}^{1} \cdot a_{i}^{0}\right) y\left(a_{i}^{1} \cdot b_{l}^{0}\right) b_{l}^{1}\right),
\end{aligned}
$$


and now (2.7) and (2.8) apply to see that $p(x *[y, z]-[x * y, z]-[y, x * z])=$ 0 .

Lemma 3.6. The map $\rho_{\mathfrak{a} X}$ in (2.15) is a Lie algebra homomorphism.

Proof. We compute

$$
\begin{aligned}
& \rho([x, y])= \rho(x \multimap y+y * x) \quad(\text { by }(3.3)) \\
&=(x \circ y+y * x)\left(a_{i}^{0}\right) a_{i}^{1}+(x \circ y+y * x)\left(b_{j}^{0}\right) b_{j}^{1} \\
&= y\left(b_{l}^{0}\right) x\left(b_{l}^{1} \cdot a_{i}^{0}\right) a_{i}^{1}+y\left(a_{k}^{0}\right) x\left(a_{k}^{1} \cdot a_{i}^{0}\right) a_{i}^{1} \\
& \quad+y\left(b_{l}^{0}\right) x\left(b_{l}^{1} \cdot b_{j}^{0}\right) b_{j}^{1}+y\left(a_{k}^{0}\right) x\left(a_{k}^{1} \cdot b_{j}^{0}\right) b_{j}^{1}, \\
& {[\rho(x), \rho(y)]=x\left(a_{i}^{0}\right) y\left(a_{k}^{0}\right)\left[a_{i}^{1}, a_{k}^{1}\right]+x\left(a_{i}^{0}\right) y\left(b_{l}^{0}\right)\left[a_{i}^{1}, b_{l}^{1}\right] } \\
& \quad+x\left(b_{j}^{0}\right) y\left(a_{k}^{0}\right)\left[b_{j}^{1}, a_{k}^{1}\right]+x\left(b_{j}^{0}\right) y\left(b_{l}^{0}\right)\left[b_{j}^{1}, b_{l}^{1}\right] \\
&=x\left(a_{k}^{1} \cdot a_{i}^{0}\right) y\left(a_{k}^{0}\right) a_{i}^{1}+x\left(b_{l}^{0}\right) y\left(b_{l}^{1} \cdot a_{i}^{0}\right) a_{i}^{1}-y\left(a_{k}^{1} \cdot b_{j}^{0}\right) x\left(a_{k}^{0}\right) b_{j}^{1} \\
& \quad+y\left(b_{j}^{0}\right) x\left(b_{j}^{1} \cdot a_{k}^{0}\right) a_{k}^{1}+x\left(a_{k}^{1} \cdot b_{j}^{0}\right) y\left(a_{k}^{0}\right) b_{j}^{1}-x\left(b_{j}^{0}\right) y\left(b_{j}^{1} \cdot a_{k}^{0}\right) a_{k}^{1} \\
& \quad-x\left(a_{i}^{1} \cdot b_{l}^{0}\right) y\left(a_{i}^{0}\right) b_{l}^{1}-x\left(b_{j}^{0}\right) y\left(b_{j}^{1} \cdot b_{l}^{0}\right) b_{l}^{1} \\
&=x\left(a_{k}^{1} \cdot a_{i}^{0}\right) y\left(a_{k}^{0}\right) a_{i}^{1}-y\left(a_{k}^{1} \cdot b_{j}^{0}\right) x\left(a_{k}^{0}\right) b_{j}^{1} \\
& \quad+y\left(b_{j}^{0}\right) x\left(b_{j}^{1} \cdot a_{k}^{0}\right) a_{k}^{1}-x\left(b_{j}^{0}\right) y\left(b_{j}^{1} \cdot b_{l}^{0}\right) b_{l}^{1},
\end{aligned}
$$

and, clearly,

$$
\begin{array}{r}
y\left(b_{l}^{0}\right) x\left(b_{l}^{1} \cdot b_{j}^{0}\right) b_{j}^{1}+y\left(a_{k}^{0}\right) x\left(a_{k}^{1} \cdot b_{j}^{0}\right) b_{j}^{1}+y\left(a_{k}^{1} \cdot b_{j}^{0}\right) x\left(a_{k}^{0}\right) b_{j}^{1}+x\left(b_{j}^{0}\right) y\left(b_{j}^{1} \cdot b_{l}^{0}\right) b_{l}^{1} \\
=([x, y]+[y, x])\left(b_{j}^{0}\right) b_{j}^{1}=0 .
\end{array}
$$

Lemma 3.7. The map $r$ defined in (2.16) is a geometric solution of the CYBE.

Proof. We must prove that $r$ satisfies (2.7), (2.8) and (2.9). To prove (2.7) we evaluate the second and third tensorand in each term at points $b, c \in X$. Let $\widetilde{\mathfrak{g}}=\mathfrak{g}_{1}+\mathfrak{g}_{2}$ and let $\left\{x_{i}\right\},\left\{x^{i}\right\}$ be dual bases of $\widetilde{\mathfrak{g}}$ and $\widetilde{\mathfrak{g}}^{*}$. In order to make formulas more readable, we call $\left\{y_{j}\right\},\left\{y^{j}\right\}$ another copy of the dual bases.

We identify vector spaces with their tangent spaces. We have:

$$
\begin{aligned}
& {\left[a_{i}^{1}, a_{k}^{1}\right] \otimes a_{i}^{0} \otimes a_{k}^{0} \mapsto[}\left.x_{i}, y_{j}\right] x^{i}(\alpha(b)) y^{j}(\alpha(c))=[\alpha(b), \alpha(c)], \\
&-a_{i}^{1} \otimes a_{k}^{1} \cdot a_{i}^{0} \otimes a_{k}^{0} \mapsto-x_{i}\left(y_{j} \cdot\left(x^{i} \alpha\right)\right)(b)\left(y^{j} \alpha\right)(c)=-x_{i}\left(\alpha(c) \cdot\left(x^{i} \alpha\right)\right)(b) \\
&=-x_{i} \alpha_{* b}(\alpha(c))\left(x^{i}\right)=-\alpha_{* b}(\alpha(c)), \\
&-a_{i}^{1} \otimes b_{l}^{0} \otimes b_{l}^{1} \cdot a_{i}^{0} \mapsto-x_{i}\left(y^{j} \beta\right)(b)\left(y_{j} \cdot\left(x^{i} \alpha\right)\right)(c)=\cdots=-\alpha_{* c}(\beta(b)) .
\end{aligned}
$$


Now, $\Psi_{\mathfrak{a}}$ is $\mathfrak{a} \rtimes \mathfrak{g}$-invariant, which means that

$$
\begin{aligned}
-\alpha_{* b}(\alpha(c)) & =-\left.\left(\rho i \pi^{-1}\right)_{*}\left(\Psi_{\mathfrak{a}}\right)_{*}\right|_{b}\left(\rho\left(i \pi^{-1} \Psi_{\mathfrak{a}}(c)\right)\right) \\
& =\left(\rho i \pi^{-1}\right)_{*}\left(\left(i \pi^{-1} \Psi_{\mathfrak{a}}(c)\right) \cdot\left(\Psi_{\mathfrak{a}}\right)(b)\right) \\
& =\rho\left(\pi^{-1}\left(\left(\pi^{-1} \Psi_{\mathfrak{a}}(c)\right) \cdot\left(\Psi_{\mathfrak{a}}(b)\right)\right), 0\right) .
\end{aligned}
$$

Analogously, setting for brevity $\tilde{b}=\Psi_{\mathfrak{a}} b, \tilde{c}=\Psi_{\mathfrak{a}} c$, we have

$$
\begin{aligned}
-\alpha_{* c}(\beta(b)) & =-\rho\left(\pi^{-1}\left(\left(\pi^{-1} \tilde{b}\right) \cdot \tilde{c}+[\tilde{b}, \tilde{c}]\right), 0\right), \\
{[\alpha(b), \alpha(c)] } & =\rho\left(\left[\pi^{-1} \tilde{b}, \pi^{-1} \tilde{c}\right], 0\right),
\end{aligned}
$$

and thus

$$
\begin{aligned}
(2.7) & =\rho\left(\pi^{-1}\left(\left(\pi^{-1} \tilde{c}\right) \cdot \tilde{b}\right)-\pi^{-1}\left(\left(\pi^{-1} \tilde{b}\right) \cdot(\tilde{c})-[\tilde{b}, \tilde{c}]\right)+\left[\pi^{-1} \tilde{b}, \pi^{-1} \tilde{c}\right], 0\right) \\
& =\rho\left(-\pi^{-1}\left(-\left(\pi^{-1} \tilde{c}\right) \cdot \tilde{b}+\left(\pi^{-1} \tilde{b}\right) \cdot(\tilde{c})+[\tilde{b}, \tilde{c}]\right)+\left[\pi^{-1} \tilde{b}, \pi^{-1} \tilde{c}\right], 0\right) \\
& =0 \quad \text { by }(2.12) .
\end{aligned}
$$

Analogously, evaluating (2.8) at $a, c$ and (2.9) at $a, b$, we get

$$
\begin{aligned}
-a_{k}^{1} \cdot b_{j}^{0} \otimes b_{j}^{1} \otimes a_{k}^{0} \mapsto & -\left(y_{j} \cdot\left(x^{i} \beta\right)\right)(a) y^{j}(\alpha(c)) x_{i}=-\beta_{* a}(\alpha(c)) \\
& \left.=\rho\left(-\pi^{-1}\left(\pi^{-1}(\tilde{c}) \cdot \tilde{a}\right),-\pi^{-1}(\tilde{c}) \cdot \tilde{a}\right)\right), \\
b_{j}^{0} \otimes\left[b_{j}^{1}, a_{k}^{1}\right] \otimes a_{k}^{0} \mapsto & x^{i}(\beta(a)) y^{j}(\alpha(c))\left[x_{i}, y_{j}\right]=[\beta(a), \alpha(c)] \\
& =\rho\left(-\left[\pi^{-1} \tilde{a}, \pi^{-1} \tilde{c}\right], \pi^{-1}(\tilde{c}) \cdot \tilde{a}\right), \\
b_{j}^{0} \otimes a_{k}^{1} \otimes b_{j}^{1} \cdot a_{k}^{0} \mapsto & x^{i}(\beta(a))\left(x_{i} \cdot y^{j} \alpha\right)(c) y_{j}=\alpha_{* c}(\beta(a)) \\
& \left.=\rho\left(\pi^{-1}\left(\pi^{-1}(\tilde{a}) \cdot \tilde{c}\right)+\pi^{-1}[\tilde{a}, \tilde{c}]\right), 0\right), \\
a_{i}^{1} \cdot b_{l}^{0} \otimes a_{i}^{0} \otimes b_{l}^{1} \mapsto & \left.x_{i} \cdot y^{j} \beta\right)(a) x^{i}(\alpha(b)) y_{j}=\beta_{* a}(\alpha(b)) \\
& \left.=\rho\left(\pi^{-1}\left(\pi^{-1}(\tilde{b}) \cdot \tilde{a}\right), \pi^{-1}(\tilde{b}) \cdot \tilde{a}\right)\right) \\
b_{j}^{0} \otimes b_{j}^{1} \cdot b_{l}^{0} \otimes b_{l}^{1} \mapsto x^{i}(\beta(a))\left(x_{i} \cdot y^{j} \beta\right)(b) y_{j}=\beta_{* b}(\beta(a)) & =\rho\left(-\pi^{-1}\left(\pi^{-1}(\tilde{a}) \cdot \tilde{b}\right)-\pi^{-1}[\tilde{a}, \tilde{b}],-\left(\pi^{-1}(\tilde{a}) \cdot \tilde{b}\right)-[\tilde{a}, \tilde{b}]\right), \\
b_{j}^{0} \otimes b_{l}^{0} \otimes\left[b_{j}^{1}, b_{l}^{1}\right] \mapsto & x^{i}(\beta(a)) y^{j}(\beta(b))\left[x_{i}, y_{j}\right]=[\beta(a), \beta(b)] \\
& =\rho\left(\left[\pi^{-1}(\tilde{a}), \pi^{-1}(\tilde{b})\right],\left(\pi^{-1}(\tilde{a}) \cdot \tilde{b}\right)-\left(\pi^{-1}(\tilde{b}) \cdot \tilde{a}\right)+[\tilde{a}, \tilde{b}]\right),
\end{aligned}
$$

and we get (2.8) and (2.9) as a result of (2.12).

\section{Proof of Theorem 1.1}

Proof. Let $r$ be a geometric solution of the CYBE. Theorem 2.5 attaches to $r$ a classical bijective cocycle 7 -tuple $\left(\mathfrak{g}, \mathfrak{a}, X, \rho_{\mathfrak{g a}}, \rho_{\mathfrak{g a} X}, \pi, \Psi_{\mathfrak{a}}\right)$. We will now "exponentiate" this classical 7-tuple to produce a (formal) quantum bijective cocycle 7-tuple. 
Recall that the category of (complex) Lie algebras is equivalent to the category of (complex) formal groups, via the exponentiation functor $\mathfrak{h} \rightarrow e^{\mathfrak{h}}$. The formal group $e^{\mathfrak{h}}$ is a scheme which can be evaluated on pro-Artinian local complex algebras; we will use, however, only $e^{\mathfrak{h}}(\mathbb{C}[[\hbar]])=\left\{e^{\Sigma}{ }_{n>0} a_{n} \hbar^{n} \mid a_{n} \in \mathfrak{h} \forall n\right\}$, and we drop $(\mathbb{C}[[\hbar]])$ from the notation. Thus we may consider the formal groups $G=e^{\mathfrak{g}}, A=e^{\mathfrak{a}}$.

To exponentiate $\rho_{\mathfrak{g a}}$, we notice that $\rho_{\mathfrak{g a}}: \mathfrak{g} \rightarrow \operatorname{Der}(\mathfrak{a})$ is a homomorphism. Hence we have a homomorphism $e^{\rho_{\mathfrak{g} \mathfrak{a}}}: e^{\mathfrak{g}} \rightarrow e^{\operatorname{Der}(\mathfrak{a})} \subseteq \operatorname{Aut}\left(e^{\mathfrak{a}}\right)$. For $\rho_{\mathfrak{g a} X}$, we have a homomorphism $\rho_{\mathfrak{g a} X}: \mathfrak{a} \rtimes \mathfrak{g} \rightarrow \operatorname{Vect}(X)$, hence we have a homomorphism $e^{\rho_{\mathfrak{g} \mathfrak{a} X}}: e^{\mathfrak{a} \rtimes \mathfrak{g}} \rightarrow e^{\operatorname{Vect}(X)}=\operatorname{Aut}(X)$, where Aut $(X)$ stands for the group of formal diffeomorphisms $X \rightarrow X$.

We have the following short exact sequence of Lie algebras: $0 \rightarrow \mathfrak{a} \rightarrow \mathfrak{a} \rtimes \mathfrak{g} \rightarrow$ $\mathfrak{g} \rightarrow 0$. By exponentiation, it maps to $1 \rightarrow e^{\mathfrak{a}} \rightarrow e^{\mathfrak{a} \rtimes \mathfrak{g}} \rightarrow e^{\mathfrak{g}} \rightarrow 1$. Since the former sequence splits, the latter also does. This gives an isomorphism $e^{\mathfrak{a} \rtimes \mathfrak{g}} \simeq e^{\mathfrak{a}} \rtimes e^{\mathfrak{g}}$. Using this isomorphism we can consider $e^{\rho_{\mathfrak{g} \mathfrak{a} X}}$ to be a map $A \rtimes G \rightarrow \operatorname{Aut}(X)$.

Consider now the map $\pi: \mathfrak{g} \rightarrow \mathfrak{a}$. This is a 1-cocycle and hence yields a Lie algebra map $\bar{\pi}: \mathfrak{g} \rightarrow \mathfrak{a} \rtimes \mathfrak{g}, x \mapsto(\pi(x), x)$. We now exponentiate it and we get $e^{\bar{\pi}}: e^{\mathfrak{g}} \rightarrow e^{\mathfrak{a} \rtimes \mathfrak{g}}$ and via the previous isomorphism we get a map $e^{\mathfrak{g}} \rightarrow e^{\mathfrak{a}} \rtimes e^{\mathfrak{g}}$. This is a morphism of groups, and it must be of the form $e^{g} \mapsto\left(\widetilde{\pi}\left(e^{g}\right), e^{g}\right)$, for some $\tilde{\pi}: e^{\mathfrak{g}} \rightarrow e^{\mathfrak{a}}$, which, a fortiori, is a 1-cocycle. Last, take $\widetilde{\Psi}_{\mathfrak{a}}=e^{\hbar \Psi_{\mathfrak{a}}}$, i.e., $\widetilde{\Psi}_{\mathfrak{a}}(x)=e^{\hbar \Psi_{\mathfrak{a}}(x)}$.

We have to prove that this is a bijective cocycle 7-tuple. It is easy to see that $\widetilde{\Psi}_{\mathfrak{a}}$ is $A \rtimes G$-equivariant; the rest of the conditions are clear.

Now define $R$ by formula (2.3). From Lemma 2.2 we know that $R$ is a solution of the quantum Yang-Baxter equation, and it is evident from the construction that it is geometric. To see that $r$ is the classical limit of $R$, we compute the first order approximation of (2.3). We can see that $x \check{*} y \equiv x-\hbar(y * x)$ and $x$ ǒ $y \equiv y-\hbar(y \multimap x)$ modulo $\hbar^{2}$. Therefore,

$$
\begin{aligned}
(R(f \otimes g))(x, y) & =(f \otimes g) R^{-1}(x, y) \\
& \equiv(f \otimes g)(x+\hbar(y * x), y+\hbar(y \multimap x))\left(\bmod \hbar^{2}\right) \\
& =f(x) g(y)+\hbar\left(f(x) b_{i}^{0}(x)\left(b_{i}^{1} \cdot g\right)(y)+\left(a_{j}^{1} \cdot f\right)(x) a_{j}^{0}(y) g(y)\right),
\end{aligned}
$$

whence $R=1+\hbar r+\mathcal{O}\left(\hbar^{2}\right)$.

Remark 4.1 (Special cases). Let us point out two special cases of this construction. The first one is when $r$ is unitary $\left(r^{21}+r=0\right)$. In this case our construction coincides with the one in [ES]; in particular, we get $R$ unitary $\left(R^{21} R=1\right)$. On the CBCST side, the property of being unitary is equivalent to $\mathfrak{a}$ being abelian, and it can be shown that when this happens $\tilde{\pi}\left(e^{g}\right)=e^{\frac{e^{g}-1}{g} \pi(g)}$ (this formula appears in [ES]). The other special case is $r$ being a classical rack $\left(r=b_{j}^{0} \otimes b_{j}^{1}\right)$. In this case we obtain a geometric rack, $R(x, y)=(x, x \circ y)$ for a suitable o (see for instance $[\mathrm{FR}]$ for the definition of rack). 


\section{Example}

In this section we apply the previous procedure to a 3-dimensional example. Let $X=\mathbb{C}^{3}=\left\{\left(x_{1}, x_{2}, x_{3}\right)\right\}, r=r^{1}+r^{2}$, where $r_{1}=\sum x_{i} \otimes A_{i}, r_{2}=\sum B_{i} \otimes x_{i}$. Here $x_{i}$ stands for the canonical coordinate function and $A_{i}, B_{i}$ are the vector fields defined by:

$$
\begin{aligned}
A_{1}(x, y, z)=\left(-\frac{x}{2}+y\right) \partial_{x}-\frac{z}{2} \partial_{z}, & B_{1}(x, y, z)=\left(-\frac{x}{2}+y\right) \partial_{x}+\left(y-\frac{z}{2}\right) \partial_{z} \\
A_{2}(x, y, z)=-x \partial_{x}+y \partial_{y}, \quad & B_{2}(x, y, z)=-x \partial_{x}+y \partial_{y}-x \partial_{z} \\
A_{3}(x, y, z)=x \partial_{x}+z \partial_{z}, \quad & B_{3}(x, y, z)=x \partial_{x}+z \partial_{z} .
\end{aligned}
$$

Following the definitions in $\S 2$, we see that $\mathfrak{a}$ can be identified with the Heisenberg algebra

$$
\mathfrak{a}=\operatorname{span}\{X, Y, C\}, \quad C \text { is in the center and }[X, Y]=C,
$$

and $\mathfrak{g}$ can be identified with the upper-triangular matrices in $\mathfrak{g l}_{2}(\mathbb{C})$,

$$
\mathfrak{g}=\left\{\left(\begin{array}{cc}
p & q \\
0 & r
\end{array}\right)\right\} .
$$

The map $\Psi: X \rightarrow \mathfrak{a}$ is just the "identity" $\Psi\left(x_{1}, x_{2}, x_{3}\right)=x_{1} X+x_{2} Y+x_{3} C$ and thus we will just identify $X$ with $\mathfrak{a}$. The action $\rho_{\mathfrak{g a}}$ is given by left multiplication by the matrix $\rho_{\mathfrak{g a}}\left(\left(\begin{array}{cc}p & q \\ 0 & r\end{array}\right)\right)=\left(\begin{array}{ccc}p & q & 0 \\ 0 & r & 0 \\ 0 & 0 & p+r\end{array}\right)$ in the basis $\{X, Y, C\}$. The 1-cocycle is given by $\pi\left(\left(\begin{array}{cc}p & q \\ 0 & r\end{array}\right)\right)=q X+r Y+\left(p+\frac{q}{2}+r\right) C$.

In particular, the adjoint action of $\mathfrak{a} \rtimes \mathfrak{g}$ on itself is given by

$$
\operatorname{adj}\left(a X+b Y+c C,\left(\begin{array}{cc}
p & q \\
0 & r
\end{array}\right)\right)=\left(\begin{array}{cccccc}
p & q & 0 & -a & -b & 0 \\
0 & r & 0 & 0 & 0 & -b \\
-b & a & p+r & -c & 0 & -c \\
0 & 0 & 0 & 0 & 0 & 0 \\
0 & 0 & 0 & -q & p-r & q \\
0 & 0 & 0 & 0 & 0 & 0
\end{array}\right)
$$

Considering the adjoint representation, which is faithful, we can compute $\widetilde{\pi}$ as



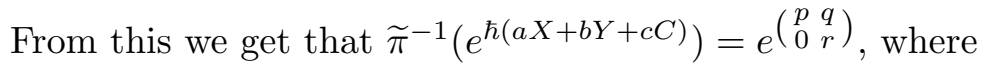

$$
\begin{aligned}
& p=\ln \left(\frac{1+\hbar c-\frac{1}{2} \hbar a}{1+\hbar b}\right) \\
& q=\hbar a(1+\hbar b) \ln \left(\frac{1+\hbar c-\frac{1}{2} \hbar a}{(1+\hbar b)^{2}}\right)\left(1+\hbar c-\frac{1}{2} \hbar a-(1+\hbar b)^{2}\right)^{-1} \\
& r=\ln (1+\hbar b) .
\end{aligned}
$$

This computation, as well as most of the remaining ones, were carried with the help of MAPLE. Finally, we can compute in a straightforward way $R(x, y)=$ 
$(x \check{*} y, x \check{y} y)$ as defined in $(2.3)$, and we get

$$
\begin{gathered}
\left(x_{1}, x_{2}, x_{3}\right) \check{*}\left(y_{1}, y_{2}, y_{3}\right)= \\
\left(\frac{1-\hbar y_{3}+\hbar \frac{y_{1}}{2}}{1-\hbar y_{2}} x_{1}-\hbar y_{1} x_{2},\left(1-\hbar y_{2}\right) x_{2},\left(1-\hbar y_{3}+\hbar \frac{y_{1}}{2}\right) x_{3}\right) ; \\
\left(x_{1}, x_{2}, x_{3}\right) \check{\circ}\left(y_{1}, y_{2}, y_{3}\right)= \\
\left(\frac{y_{1}\left(1-\hbar x_{2}\right)+\hbar y_{2}\left(x_{1}-y_{1}+\hbar y_{1} x_{2}-\hbar x_{1} y_{3}+\frac{1}{2} \hbar x_{1} y_{1}\right)}{\operatorname{den}}, \frac{y_{2}}{\left(1-\hbar x_{2}+\hbar^{2} x_{2} y_{2}\right)},\right. \\
\left.\frac{\left(1-\hbar y_{2}\right)\left(y_{3}-\hbar y_{1} x_{2}\right)+\hbar y_{2} x_{1}\left(1-\hbar y_{3}+\frac{1}{2} \hbar y_{1}\right)}{\operatorname{den}}\right)
\end{gathered}
$$

where

$$
\text { den }=\left(1-\hbar y_{2}\right)\left(1-\frac{1}{2} \hbar^{2} y_{1} x_{2}\right)+\hbar\left(1-\hbar y_{3}+\frac{1}{2} \hbar y_{1}\right)\left(-x_{3}+\frac{1}{2} x_{1}+\hbar x_{3} y_{2}\right) .
$$

It is possible to "unitarize" this example by replacing in $\mathfrak{a}$ the bracket $[X, Y]=$ $C$ by $[X, Y]=\varepsilon C$. The r-matrix $r^{\varepsilon}$ has a similar expresion to that of $r$, but changing $A_{i}, B_{i}$ by

$$
\begin{aligned}
A_{1}^{\varepsilon}(x, y, z)=\left(-\varepsilon \frac{x}{2}+y\right) \partial_{x}-\varepsilon \frac{z}{2} \partial_{z}, & B_{1}^{\varepsilon}(x, y, z)=\left(-\varepsilon \frac{x}{2}+y\right) \partial_{x}+\varepsilon\left(y-\frac{z}{2}\right) \partial_{z}, \\
A_{2}^{\varepsilon}(x, y, z)=-x \partial_{x}+y \partial_{y}, & B_{2}^{\varepsilon}(x, y, z)=-x \partial_{x}+y \partial_{y}-\varepsilon x \partial_{z}, \\
A_{3}^{\varepsilon}(x, y, z)=x \partial_{x}+z \partial_{z}, & B_{3}^{\varepsilon}(x, y, z)=x \partial_{x}+z \partial_{z} .
\end{aligned}
$$

The 1-cocycle will become $\pi^{\varepsilon}\left(\left(\begin{array}{cc}p & q \\ 0 & r\end{array}\right)\right)=q X+r Y+\left(p+\frac{\varepsilon q}{2}+r\right) C$. There are similar expresions for the R-matrix $R^{\varepsilon}$. Now, if we let $\varepsilon \rightarrow 0$, we will get a unitary r-matrix (since $\mathfrak{a}$ will become abelian) and hence a unitary R-matrix. In the limit, we get $\lim _{\varepsilon \rightarrow 0} R^{\varepsilon}(x, y)=\left(x \check{\varkappa}^{0} y, x \check{\circ}^{0} y\right)$, given by

$$
\begin{aligned}
& \left(x_{1}, x_{2}, x_{3}\right) \check{\varkappa}^{0}\left(y_{1}, y_{2}, y_{3}\right)=\left(\frac{1-\hbar y_{3}}{1-\hbar y_{2}} x_{1}-\hbar y_{1} x_{1},\left(1-\hbar y_{2}\right) x_{2},\left(1-\hbar y_{3}\right) x_{3}\right) \\
& \left(x_{1}, x_{2}, x_{3}\right) \check{\circ}^{0}\left(y_{1}, y_{2}, y_{3}\right)= \\
& \left(y_{1} \frac{1-\hbar x_{2}}{1-\hbar x_{3}+\hbar^{2} x_{3} y_{3}}\right. \\
& \quad+\hbar y_{2} \frac{x_{1}\left(1-\hbar y_{3}\right)}{\left.1-\hbar x_{3}+\hbar^{2} x_{3} y_{3}\right)\left(1-\hbar y_{2}\right)}, \frac{y_{2}}{1-\hbar x_{2}+\hbar^{2} x_{2} y_{2}}, \\
& \left.\quad \frac{y_{3}}{\left.1-\hbar x_{3}+\hbar^{2} x_{3} y_{3}\right)}\right)
\end{aligned}
$$

\section{References}

[D] V. Drinfeld, Some unsolved problems in quantum group theory, Quantum groups (Leningrad, 1990), 1-8, Lecture Notes in Math., 1510, Springer, Berlin, 1992.

[EK] P. Etingof, D. Kazhdan, Quantization of Lie bialgebras. I, Selecta Math. (N.S.) 2 (1996), 1-41. 
[ES] P. Etingof, A. Soloviev, Quantization of geometric classical r-matrices, Math. Res. Lett. 6 (1999), 223-228.

[ESS] P. Etingof, T. Schedler, A. Soloviev, Set-theoretical solutions to the quantum YangBaxter equation, Duke Math. J. 100 (1999), 169-209.

[FR] R. Fenn, C. Rourke, Racks and links in codimension two, J. Knot Theory Ramifications 1 (1992), 343-406.

[S] A. Soloviev, Non-unitary set-theoretical solutions to the quantum Yang-Baxter equation, Math. Res. Lett. 7 (2000), 577-596.

Pavel Etingof:

Mit Math. Dept. Of. 2-176

77 Mass. Ave.

02139, CAMBridge, MA, USA.

E-mail address: etingof@math.mit.edu

Matías Graña:

Mit Math. Dept. Of. 2-155

77 Mass. Ave.

02139, CAMBridge, MA, USA.

Permanent:

Depto. Matemática - FCEyN - UBA.

PAB. I - CiUdad Universitaria.

(1428) Ciudad de Buenos Aires - Argentina

E-mail address: matiasg@math.mit.edu 